\title{
Airborne radiometric validation of the geostationary lightning mapper using the Fly's Eye GLM Simulator
}

\author{
Mason G. Quick ${ }^{a}, *, \dagger$ Hugh J. Christian, ${ }^{b}$ Katrina S. Virts, ${ }^{\text {b }}$ and \\ Richard J. Blakeslee ${ }^{a}$ \\ ${ }^{a}$ NASA Marshall Space Flight Center, National Space Science and Technology Center, \\ Huntsville, Alabama, United States \\ ${ }^{b}$ University of Alabama in Huntsville, National Space Science and Technology Center, \\ Huntsville, Alabama, United States
}

\begin{abstract}
The Fly's Eye GLM Simulator (FEGS) is a multi-spectral array of radiometers designed to provide a validation dataset for the geostationary lightning mapper (GLM). The main component of FEGS is a $5 \times 5$ grid of radiometers, each with a square 18 deg field of view, that are sensitive to a 10-nm wide spectral band centered on $780 \mathrm{~nm}$ to observe a neutral atomic oxygen emission triplet at $777.4 \mathrm{~nm}$. FEGS was flown as a primary payload on the highaltitude NASA ER-2 aircraft as part of the GOES-R validation flight campaign in the spring of 2017. More than 14,000 lightning flashes were recorded over a variety of thunderstorm phenomenologies and geographical regions in the continental United States and Atlantic Ocean. Following the application of a quality control process, a subset of 9160 of these flashes occurring during daylight hours was suitable for analysis. This analysis has provided an independent, lower bound estimate of the average daytime optical flash detection efficiency (FDE) of GLM at $64 \%$. The FDE varied significantly between storms and location ranging between $44 \%$ and $88 \%$. An upper estimate of the time-integrated cloud-top radiance detection threshold of GLM during daytime observations is calculated to be $9 \mu \mathrm{J} / \mathrm{m}^{2} \mathrm{srnm}$. Dependencies of GLM performance on the temporal width, energy, and cloud-top area of optical pulses, and the background solar radiance conditions are presented and discussed. (C) The Authors. Published by SPIE under a Creative Commons Attribution 4.0 Unported License. Distribution or reproduction of this work in whole or in part requires full attribution of the original publication, including its DOI. [DOI: 10.1117/1.JRS.14.044518]
\end{abstract}

Keywords: geostationary lightning mapper; validation; calibration; lightning; aircraft; radiometry; thunderstorms; optical; remote sensing; field campaign; cloud top; high altitude; optical emission.

Paper 200607 received Aug. 21, 2020; accepted for publication Nov. 13, 2020; published online Dec. 9, 2020.

\section{Introduction}

Observations of the optical signatures of lightning that are radiated through the top of clouds have been previously collected from high-altitude aircraft to quantify the temporal, spectral, and energetic characteristics of lightning emissions. These measurements have been used to inform the design requirements of space-based lightning imagers and to investigate lightning physics. An extensive set of observations were recorded in the early 1980s from the high-altitude NASA U-2 aircraft. The effort was undertaken to better quantify performance specifications of an envisioned future geostationary lightning imager. ${ }^{1-5}$

Christian et al. $^{3}$ described a suite of sensors that were integrated onto the U-2 aircraft to observe both the optical and electrical characteristics of lightning from an altitude of 20 to $22 \mathrm{~km}$. It included a photoelectric radiometer called the optical pulse sensor (OPS) with a wide (60 deg) field of view (FOV) and exchangeable spectral bandpass filters that were used to isolate

\footnotetext{
*Address all correspondence to Mason G. Quick, mgquick@umd.edu

${ }^{\dagger}$ Present Address: University of Maryland, Earth System Science Interdisciplinary Center, College Park, Maryland, United States
} 
the lightning generated near-infrared emission lines from either neutral atomic oxygen (OI, $777.4 \mathrm{~nm}$ ) or neutral atomic nitrogen (NI, $868.3 \mathrm{~nm}$ ). It also included a broadband spectrometer that recorded the lightning spectrum with $2 \mathrm{~nm}$ spectral resolution between 400 and $900 \mathrm{~nm}$ and with $5 \mathrm{~ms}$ temporal resolution. The study measured 79 lightning flashes containing over 1200 optical pulses and reported waveforms, spectra, and frequency distributions of pulse and flash parameters. The dual optical pulse sensor (DOPS) was a two-channel radiometer with matching $60 \mathrm{deg}$ FOV that was developed and flown on later U-2 flights.

Measurements collected during the U-2 flights described above were used to inform the sensor design requirements and estimate the postlaunch detection efficiency of three space-based lightning imagers in low earth orbit. These imagers included the optical transient detector onboard MICROLAB-1 that operated from 1995 to $2000,{ }^{6}$ the lightning imaging sensor (LIS) onboard the Tropical Rainfall Measuring Mission (TRMM) satellite that operated from 1997 to $2015,^{7,8}$ as well as the LIS duplicate flight model onboard the International Space Station from 2017 to present. ${ }^{9,10}$

In August 2002, a second major airborne measurement of lightning optical emissions was collected as part of the Altus Cumulus Electrification Study. ${ }^{11}$ This study employed the Altus II unmanned aerial vehicle (UAV), which flew at an altitude of 15 to $17 \mathrm{~km}$ with the OPS and DOPS from the previous U-2 campaign. Two spectral bandpass filters were installed to observe OI and ionized atomic nitrogen (NII, $500 \mathrm{~nm}$ ) simultaneously. The single-channel OPS was mounted to view 45 deg downward out of the side of the UAV while observing the 777.4 spectral band, and the DOPS viewed at nadir. This study measured 587 lightning flashes containing 5256 optical pulses, significantly increasing the population of cloud-top optical data. Frequency distributions and statistical analysis of flash and pulse parameters are reported in Ref. 11.

With the launch of the geostationary lightning mapper (GLM) onboard the National Oceanic and Atmospheric Administration's (NOAA) Geostationary Operational and Environmental Satellite (GOES-R series), the realization of an optical lightning sensor in geostationary orbit has been achieved. ${ }^{12,13}$ GLM onboard the GOES-East and GOES-West platforms provide continuous lightning optical imaging for the entire lifecycle of thunderstorms across the Americas and the Pacific and Atlantic oceans.

A large validation effort has been undertaken for the GOES-R series because it constitutes a major upgrade in the NOAA Earth remote sensing technology. ${ }^{14}$ For validation of GLM, a combination of ground-based, space-based, and airborne measurement capabilities have been utilized. ${ }^{15}$ Ground-based lightning detection technologies provide a temporal and spatial dataset to asses lightning detection performance over the full GLM domain but are sensitive to radiofrequency emission that is not necessarily associated with the optically bright processes of the lightning discharge. ${ }^{16}$ For a critical assessment of radiometric performance of GLM, an airborne GLM simulator has been designed to observe the thunderstorm optical emission from above the cloud. The Fly's Eye GLM Simulator (FEGS) provides a directly comparable observation of lightning optical emission through the cloud-top, similar to that which is also achieved by comparing LIS to GLM. FEGS represents an order of magnitude improvement in capability over previous airborne radiometric observations of lightning.

\section{FEGS Instrument}

\subsection{Optomechanical Design}

FEGS is a multi-spectral array of radiometers optimized to detect the optical emission from lightning. It consists of a main array of 25 radiometers $(5 \times 5$ grid $)$, each tilted at a different viewing angle so that the individual FOVs combine to create an image of the cloud-top, acquired at a rate of $100 \mathrm{k}$ samples per second. Radiometers in the main FEGS array observe line emission from OI in the hot lightning channel at $777.4 \mathrm{~nm}^{17,18}$ using a spectral filter with a $10-\mathrm{nm}$ fullwidth at half-maximum passband and central wavelength of $780 \mathrm{~nm}$. FEGS also includes five additional nadir viewing radiometers that are sensitive to alternate spectral bands that were included to probe the physical properties of the observed discharge process. Central wavelengths of the spectral filters include 400, 500, 660, and $675 \mathrm{~nm}$, and a wideband visible and near IR 


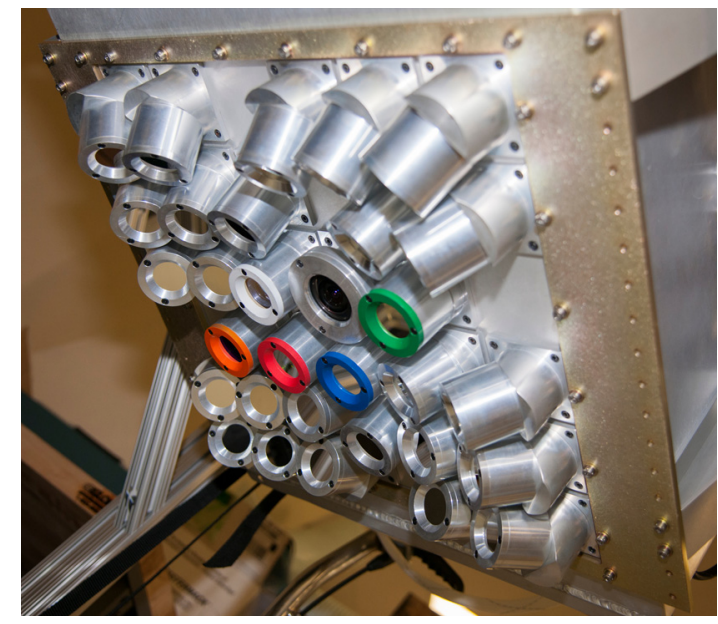

Fig. 1 A photograph of the FEGS radiometer array. Radiometers are tilted at different viewing angles to create a gridded combined FOV and an image of the cloud-top. Radiometers with colored caps indicate the channels with alternate spectral filters. The large aperture fixture near the center is the wide-angle HD camera.

filter covering 400 to $1000 \mathrm{~nm}$. A high-definition $(1080 \times 1920$ resolution) camera is also included to provide contextual high-resolution imagery of the cloud-top. In Fig. 1, the 25 radiometers in the main $5 \times 5$ array are shown mounted at various looking angles and the 5 radiometers with alternate spectral bands can be identified by mounting caps of varying color. The wide-angle camera is located near the center of the array and can be identified by its relatively large aperture. In this report, discussion will be confined to the radiometric data from the main FEGS array in the context of GLM validation.

FEGS was carried on the NASA ER-2 high-altitude aircraft, which is capable of flying above $20 \mathrm{~km}$ altitude, higher than the maximum altitude of virtually all thunderstorm systems. A geometric projection of the FEGS spatial footprint is shown in Fig. 2. This projection assumes that the aircraft is $5 \mathrm{~km}$ above a flat cloud-top, which was the nominal design condition for the instrument.

The radiometers have an individual FOV that is square with a half angle of $9.46 \mathrm{deg}$. All 30 radiometers in the FEGS array are mounted to a plate on the downward facing surface of the instrument enclosure when situated in the ER-2 aircraft. The payload was carried in the forward section of the superpod compartment mounted beneath the wing of the aircraft and viewed the storm through a standard glass window. The viewing angle of each radiometer in the main array is separated by $18 \mathrm{deg}$ in the $x$ and $y$ direction. This orientation results in minimal overlap of the individual radiometer FOV while providing full coverage within the combined FOV of the FEGS array (Fig. 2).

FEGS is packaged with a suite of instruments named the Airborne Lightning Observatory for FEGS and Terrestrial (ALOFT) gamma-ray flashes package. The payload includes FEGS, a twochannel electric field change meter (EFCM), and two scintillator instruments that detect gammaray and high-energy particle emission from thunderstorms. The ALOFT package adds to the NASA Airborne Earth Science Program the capability to investigate submillisecond lightning energetics by detecting signals radiated by lightning that range from radio frequencies to gammaray emission. ${ }^{19}$

\subsection{Pulse Detection and Radiometric Calibration}

The FEGS radiometers sample the instantaneous cloud-top radiance field and are digitized with 16-bit resolution by the front-end electronics. When run in continuous data collection mode, the 30 radiometers produce $6 \mathrm{MB}$ of data per second. To detect flashes in the continuously recorded waveforms, an algorithm was applied postflight to identify transient pulses with radiance amplitudes that exceeded a dynamic threshold value. The threshold was adjusted based on the 


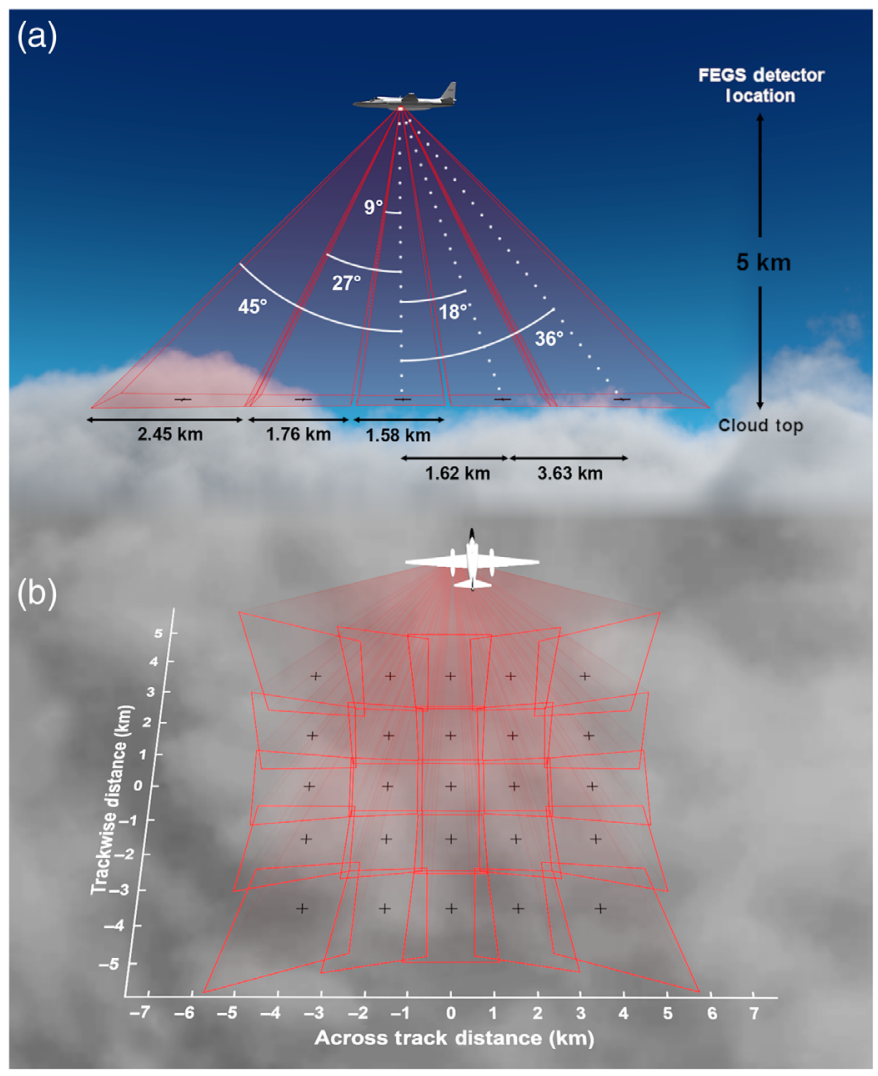

Fig. 2 (a) A cross section showing the looking angle orientation of the FEGS array with estimated spatial footprints of the individual radiometers. Dotted lines illustrate the normal vector extending along the optical axis of an individual radiometer. Solid lines illustrate the boundary of an individual radiometer FOV. (b) The projected spatial footprint of the FEGS array for a flat cloud-top. Distance estimated for the radiometer footprints assume the ER-2 is $5 \mathrm{~km}$ above the cloud-top surface.

amplitude of the random noise that was superimposed on the scene background radiance baseline. The estimated baseline was subtracted out and temporal and spatial characteristics of the lightning optical pulses were measured. Pulse detections analyzed in this report were produced by the second iteration (version 2) of the data processing algorithm. Finally, the pulses were clustered into flashes using a sliding time window of $330 \mathrm{~ms}$, the same time criterion used by the GLM flash clustering algorithm. ${ }^{20,21}$ Caveats of this definition of a flash are discussed in Sec. 5.1.

Calibration of the spectral responsivity of the FEGS radiometers was performed at the National Space Science and Technology Center. For each optical pulse observed by FEGS, the spectral radiance $L_{\lambda}\left(\mathrm{W} / \mathrm{m}^{2} \mathrm{srnm}\right)$ leaving the surface of the cloud-top as a function of time was estimated by assuming the radiance was uniform over the projected area of each pixel that was illuminated by the pulse. These estimations assume that the angular distribution of cloud-top emission is Lambertian, or constant as a function of angle. Then the time-integrated spectral radiance $\left(\mathrm{J} / \mathrm{m}^{2} \mathrm{srnm}\right)$, which in this report will be represented by $J_{\lambda}$, was estimated by summing the spectral radiance waveforms of all illuminated pixels, integrating the summed waveform over the duration of the pulse, and assuming the radiance was uniform over the total projected area of the illuminated pixels. Finally, the total $J_{\lambda}$ of each flash is calculated by summing the $J_{\lambda}$ of each pulse in the flash. Overlap of FEGS pixel coverage results in a small overestimation of pulse radiance reaching a maximum of $11 \%$ with a fully illuminated FOV. FEGS can detect illumination amplitudes well below amplitudes observable by GLM. A discussion of the FEGS sensitivity in flight is given in Sec. 4.3.

An important assumption used to quantify lightning emission in this report is that all lightning radiance within the $10-\mathrm{nm}$ passband of the $780-\mathrm{nm}$ CWL spectral filter is produced by an OI 
emission triplet that is $1 \mathrm{~nm}$ wide. Thus when computing both $L_{\lambda}$ and $J_{\lambda}$ the effective spectral bandwidth $\left(\Delta \lambda_{e}\right)$ of the FEGS radiometers is assumed to be $1 \mathrm{~nm}$. This assumption allows the FEGS radiance measurements to be readily compared to the previous literature, and its validity is discussed in Sec. 4.3.

\section{Data Collection and Quality Control}

\subsection{GOES-R Validation Flight Campaign}

From March to May of 2017 FEGS was flown on the ER-2 as the primary instrument for GLM validation. Eleven flights of the campaign were conducted to observe thunderstorms in a variety of geographical regions including the San Joaquin Valley in California, Colorado Plateau, Central Plains, Tennessee River Valley, Great Lakes, Gulf and Atlantic Coasts, and Atlantic Ocean. A map of the detected optical flash locations is shown in Fig. 3. Types of thunderstorms that were observed included isolated convection, weakly organized convection, mesoscale convective systems, quasi-linear convective systems, and supercell thunderstorms. The storms were observed during day, night, dusk, and dawn, at latitudes ranging between 27 and 44 deg north. The observed flashes reside within a limited range of satellite boresight angle, between $\sim 4.8$ to 7.1 deg out of the $\sim 8$ deg GLM FOV measured from the optical axis of the sensor. A distribution of the local hour of flash detections is shown in Fig. 4 and generally follows the temporal profile of lightning activity over land in North America. ${ }^{22}$

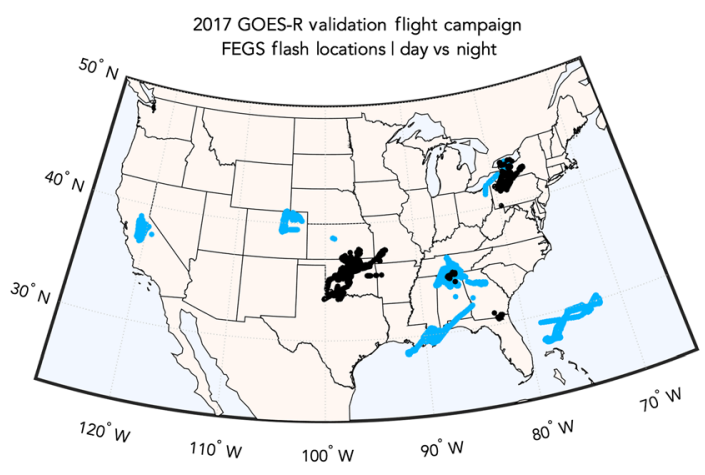

Fig. 3 Map of observed optical flashes in the Version 2 FEGS dataset. Blue circles indicate a flash detected during the daylight (local hour 6 to 18) and black circles indicate flashes detected at night.

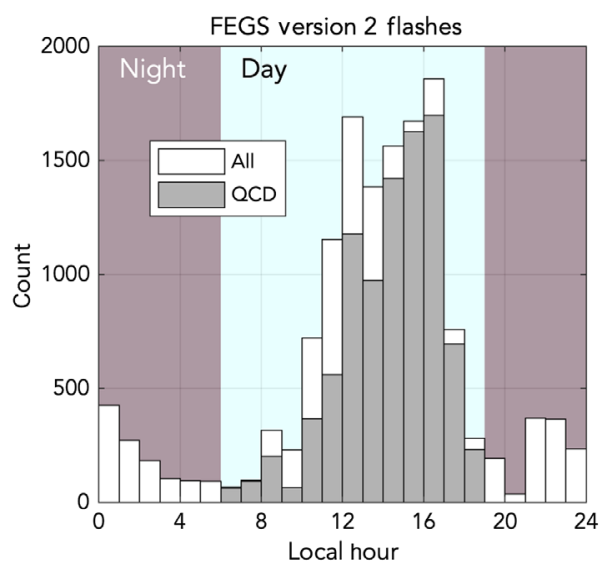

Fig. 4 Distribution of flashes by local hour for all detected FEGS flashes and for flashes that passed the QC conditional filters. 
Because the field campaign took place during the postlaunch validation phase of the GOES-R deployment, continuous modification to the GLM ground processing algorithm was occurring during and after the collection of FEGS data. To facilitate an intercomparison with the modern GLM processing algorithms, the GLM L1-b data ${ }^{20,21,23}$ for the time periods associated with the campaign flights was reprocessed to produce an L2 dataset using an updated algorithm.

\subsection{Quality Control Data Reduction}

We apply a number of quality control (QC) filters that reduce the dataset to include only pulses with good radiometric alignment and complete radiance waveforms. For radiometric alignment, we include only pulses detected when the ER-2 was in level flight at altitudes of $>15 \mathrm{~km}$ so that no pulses detected during aircraft ascent or decent are considered. Additionally, we require that the ER-2 wings were nearly flat with a roll angle of $<5$ deg to ensure the projected FOV of the radiometer array was not highly skewed with respect to the cloud-top. A small negative voltage bias at the output of the FEGS front-end amplifiers caused dark scene background radiance to fall below the smallest bit in the subsequent analog-to-digital converter. As a result, the waveforms of pulses that were observed at night were partially clipped causing inaccurate radiance measurement and non-detection of low-amplitude pulses. To remove any bias caused by this issue, we include only pulses that were recorded during daylight from local hours 6 to 18 (Fig. 4).

Finally, transients in the noise signature can occasionally pass the pulse detection algorithm as a false detection (FD). During the signal processing, any pulse that was detected in only one radiometer was discarded under the assumption that it was caused by noise. Additionally, an FD flag was introduced to identify pulses with high probability of being false. The flag was based on an identified pattern of electrical cross talk between radiometer channels on the same data system read-out chain. Some electronic transients were also flagged manually as false. Reducing the dataset in this way did not have an appreciable effect on the observed pulse radiance distribution, and the median radiance value is preserved to within 5\%. Often, a single-false transient would by itself create a false flash so that flash counts are affected more significantly by QC than pulse counts. In this paper, we analyze only optical pulses/flashes that pass all the criterion discussed above and we refer to this subset of detections as the quality controlled daytime (QCD) dataset. To assist with QC and interpretation of the optical waveforms, the FEGS dataset was intercompared with waveforms from the EFCM in the ALOFT package as well as data from two groundbased lightning location systems, namely the National Lightning Detection Network ${ }^{24}$ and the Earth Networks Total Lightning Network. ${ }^{25}$

\section{Results}

\subsection{GLM Flash Detection Efficiency}

The primary mission objective for the FEGS instrument was to determine the flash detection efficiency (FDE) of GLM. To accomplish this, the observed GLM flashes were checked against FEGS flashes using a geospatial and temporal comparison with the following criteria. First, for a match to be declared between the two datasets, a GLM flash centroid ${ }^{20}$ must be $<30 \mathrm{~km}$ offset from the location of FEGS at the time the flash was detected. Second, the duration of the GLM flash must overlap the duration of the FEGS flash in time allowing for a 500-ms window between the end of one instrument's flash and the beginning of the other. If more than one GLM flash was found to match, a FEGS flash then the GLM flash with the smallest distance offset was selected as the singular match. Any FEGS flash that did not have a matching GLM flash was considered a GLM miss. The GLM FDE is the percentage of FEGS flashes that had a corresponding GLM match.

A tabulation of the FDE for QCD flashes is shown in Table 1 for each flight of the 2017 field campaign. GLM's overall FDE is estimated to be $64 \%$ during the daytime, though there was a large variability between flights, with values ranging from $44 \%$ to $88 \%$. Analysis of the 9160 QCD flashes that were observed during the 11 flights in the field campaign does not reveal 
Table 1 FEGS Version 2 data collection summary during 2017 GOES-R Validation Flight Campaign. Flash and pulse counts include quality-controlled detections during daytime flights (QCD). Storm descriptions include Quasi-Linear Convective System (QLCS), Mesoscale Convective System (MCS), and Low Flash Rate (LFR) storms.

\begin{tabular}{|c|c|c|c|c|c|c|c|c|c|}
\hline DATE & LOCATION & STORM TYPE & DAY/NIGHT & $\begin{array}{l}\text { Flash } \\
\text { Count }\end{array}$ & $\begin{array}{l}\text { QCD } \\
\text { Flash } \\
\text { Count }\end{array}$ & $\begin{array}{l}\text { QCD } \\
\text { GLM } \\
\text { FDE }\end{array}$ & $\begin{array}{l}\text { Pulse } \\
\text { Count }\end{array}$ & $\begin{array}{l}\text { QCD } \\
\text { Pulse } \\
\text { Count }\end{array}$ & $\begin{array}{l}\text { QCD } \\
\text { GLM } \\
\text { PDE }\end{array}$ \\
\hline $03 / 21$ & California & Active QLCS & Day & 2289 & 2181 & 51 & 29934 & 28573 & 16 \\
\hline $04 / 16$ & Oklahoma & Large MCS & Night/Dawn & 390 & 61 & 85 & 1710 & 440 & 61 \\
\hline $04 / 18$ & Huntsville & Convective Initiation & Day & 348 & 316 & 44 & 2370 & 1905 & 36 \\
\hline $04 / 20$ & Toronto & Org. Convection & Dusk & 394 & 58 & 74 & 1879 & 470 & 49 \\
\hline $04 / 22$ & Huntsville & Tornadic QLCS & Day/Dusk & 2654 & 2423 & 73 & 24150 & 22269 & 37 \\
\hline $04 / 27$ & Huntsville & Horiz. Extensive LFR & Night & 39 & - & - & 634 & - & - \\
\hline $04 / 29$ & Oklahoma & Supercell & Night & 1064 & - & - & 5324 & & - \\
\hline $05 / 08$ & Colorado & Severe Hail & Day & 2454 & 2311 & 54 & 21428 & 20285 & 17 \\
\hline $05 / 12$ & Louisiana & Weak QLCS & Day & 2674 & 1262 & 88 & 16353 & 14052 & 48 \\
\hline $05 / 14$ & Atlantic/KSC & Org. Convection LFR & Day & 1234 & 548 & 74 & 7653 & 6256 & 49 \\
\hline \multirow[t]{4}{*}{$05 / 17$} & Oklahoma & Active QLCS & Night & 606 & - & - & 3778 & - & - \\
\hline & & & Day & 11781 & 9160 & 64 & 105477 & 94250 & 29 \\
\hline & & & Night & 2365 & - & - & 22311 & - & - \\
\hline & & & TOTAL & 14146 & 9160 & 64 & 115213 & 94250 & 29 \\
\hline
\end{tabular}

a statistically identifiable trend in FDE with latitude, longitude, or satellite boresight angle. We note, however, that storms in California and Colorado have 2 of the lowest 3 FDE values and correspond to the highest boresight observations. Although a dependance of FDE on boresight angle is expected due to varying GLM threshold settings, the statistical results from this analysis do not allow a determination of the relative effect of boresight angle compared to other factors such as storm phenomenology. The observed distance offset between the FEGS position and the GLM flash centroid was broadly distributed with a peak at $13 \mathrm{~km}$ and a standard deviation of $7 \mathrm{~km}$.

We identify no statistically significant correlation between FDE and background reflected solar spectral radiance in this set of observations. A histogram showing the distribution of background spectral radiance $L_{\lambda, \mathrm{bg}}\left(\mathrm{W} / \mathrm{m}^{2} \mathrm{sr} \mathrm{nm}\right)$ values at the time of FEGS flash detections is shown in Fig. 5. Note that to compute the background solar radiance, we apply the true $10 \mathrm{~nm}$ bandwidth of the FEGS spectral filter and do not integrate over time. The flash counts are separated by GLM misses and matches, and the FDE in each bin is shown as a black trace that scales with the right axis. A large spread is observed in FDE which ranges from $44 \%$ to $93 \%$. This range closely matches the range of values listed in Table 1 indicating that factors other than background radiance dominate the FDE in this dataset.

The ability of GLM to detect a lightning flash is highly dependent on the duration of the flash. Figure 6 shows GLM FDE as a function of FEGS flash duration as well as a histogram illustrating the frequency distribution of flash duration in bins of $10 \mathrm{~ms}$. The calculated GLM FDE is about $50 \%$ for flashes with a duration $<200 \mathrm{~ms}$. There is a dramatic increase in FDE for durations ranging between 200 and about $500 \mathrm{~ms}$, and flashes with duration $>500 \mathrm{~ms}$ are generally detected with high efficiency ( $>80 \%$ ). We observe an asymptotic approach toward $100 \%$ FDE as flash duration increases to $>1 \mathrm{~s}$. High statistical variability in FDE at long duration corresponds to relatively small sample populations. 


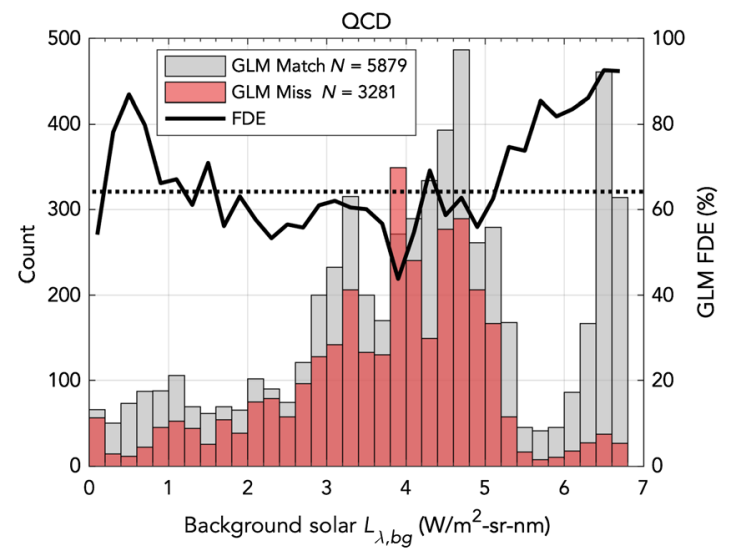

Fig. 5 GLM FDE as a function of background solar spectral radiance $L_{\lambda, \text { bg }}$. A histogram showing the number of flashes that were detected by FEGS under varying background radiance conditions is parsed into GLM misses (red) and GLM matches (gray). The histogram scales with the left axis. The FDE is calculated for each bin and is plotted as the solid black line and scales with the right axis. The overall average FDE is shown as a dotted line.

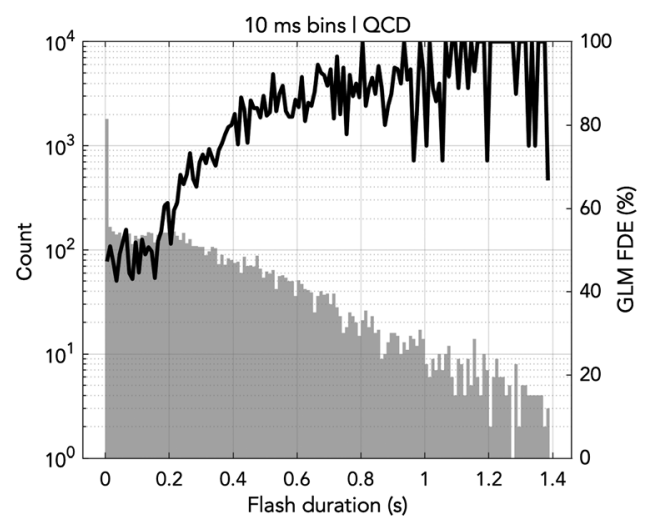

Fig. 6 GLM FDE as a function of flash duration. A histogram illustrating the number of flashes that were recorded with durations binned by $10 \mathrm{~ms}$ is shown in gray and scales with the left axis. The GLM FDE is calculated for each bin and shown as a black trace that scales with the right axis.

\subsection{GLM Subflash Performance}

Although FDE is quoted as the GLM performance specification, ${ }^{12}$ analysis of the subflash pulse detection efficiency (PDE) enables a deeper understanding of the instrument's ability to detect lightning. Here we characterize GLM's ability to detect the individual optical pulses that compose a flash. Table 1 lists the number of QCD pulses detected and the GLM PDE for the nine flights of the GOES-R validation flight campaign that included daytime observations. The overall daytime PDE of GLM is $29 \%$ with values ranging between $16 \%$ and $61 \%$ for the various flights.

The distributions of time offsets between the beginning and end of GLM and FEGS flashes are illustrated in Fig. 7. In this figure, the two left panels show the time difference between the flash beginning time and the two right panels show the offset between flash ending times with bin sizes indicated in the panel titles. Number distributions are shown as gray histograms that scale with the left axes and the corresponding cumulative distribution functions (CDF) are shown as black traces that scale with the right axes. There appear to be two populations superimposed in the distributions. The first is best observed in Figs. 7(a) and (b) with $50 \mathrm{~ms}$ bins and features relatively low-count numbers that are broadly distributed over the \pm 1 -s interval. We interpret these flashes as likely originating or ending with illumination outside of the FEGS FOV. 

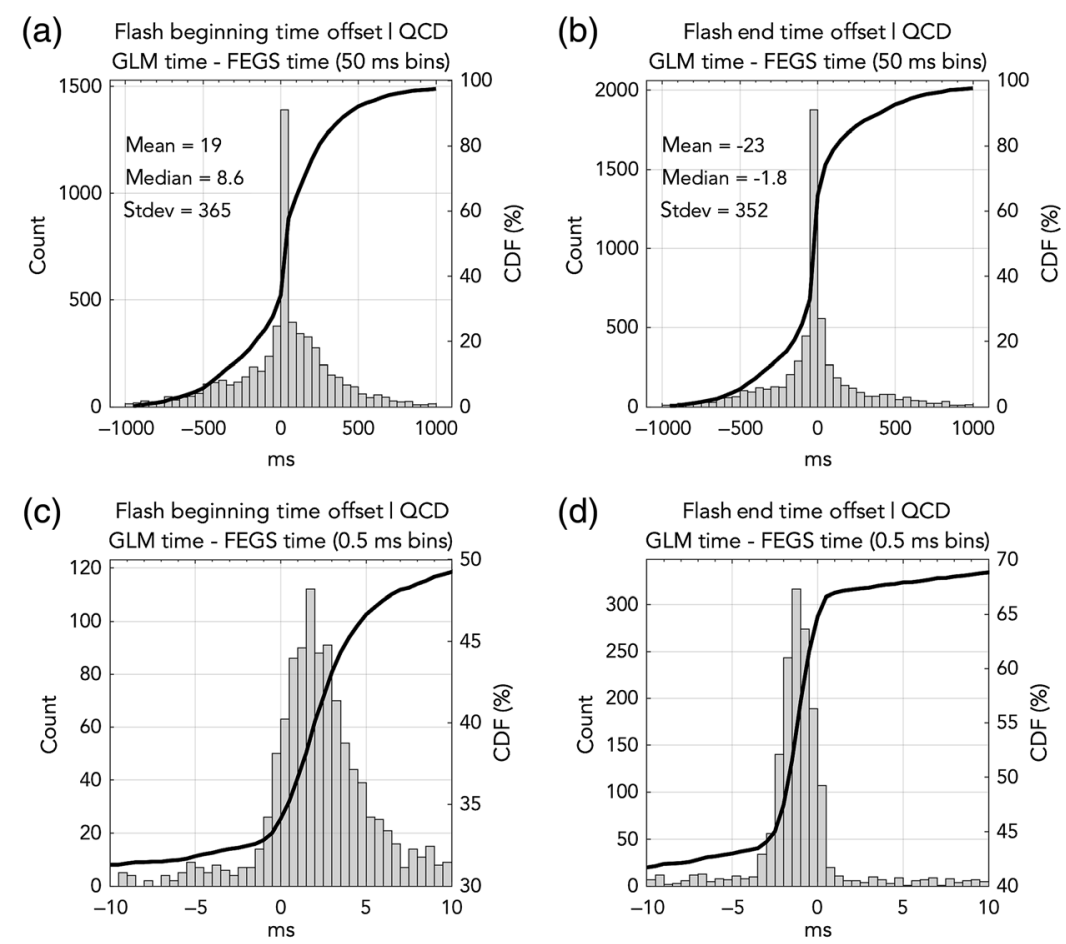

Fig. 7 Time offset between GLM and FEGS flash start and end times. Each panel shows the distribution of time offsets as a gray histogram that scales with the left axes. The corresponding CDFs are shown as a black trace that scales with the right axes. (a) Time offset between the flash beginning time using 50 ms bins; (b) time offset between the flash ending time using 50 ms bins; (c) time offset between the flash beginning time using $0.5 \mathrm{~ms}$ bins; and (d) time offset between the flash ending times using $0.5 \mathrm{~ms}$ bins.

The second population is sharply peaked with time offsets smaller than $\pm 5 \mathrm{~ms}$ and appears clearly in Figs. 7(c) and (d) using $0.5 \mathrm{~ms}$ bins. This population includes flashes where FEGS directly observed the initial illuminated area. The initiation of GLM flashes most commonly occurs after but within about $6 \mathrm{~ms}$ of the beginning of the corresponding FEGS flash with a median time offset of $8.6 \mathrm{~ms}$. There is less spread in the time offset distribution for the end of flashes where the most common occurrence is that the GLM flashes end within 4 ms prior to FEGS. The distribution has a median offset of $-1.8 \mathrm{~ms}$ where the negative sign indicates that the GLM flash ended first.

In the FEGS observations, we note that over the course of a flash the pulse radiance increases on average, leading to an increase in PDE and a corresponding increase in FDE. This is direct and independent corroboration of results found by Ref. 26 who identify similar trends in observations from the LIS on the TRMM satellite. Further implications of this trend are discussed in Sec. 5.

We also find a strong dependence of PDE on the temporal width of the optical pulses. Although in general pulses with higher $J_{\lambda}$ tend to have broader temporal widths, these two parameters exhibit $<50 \%$ correlation. Thus a dependence of PDE on pulse width has in impact on GLM PDE that is largely independent of $J_{\lambda}$. Histograms of the 10-10\% amplitude width of pulses that GLM missed and matched, along with corresponding PDE as a function of width are shown in Fig. 8. Virtually all pulses observed by FEGS that had a simple, single peaked waveform $^{11}$ and occurred in temporal isolation had $10-10 \%$ widths that were $<2 \mathrm{~ms}$. We note that pulse widths that are short compared to the 2-ms GLM integration period have a low probability of being detected, and PDE increases generally with pulse width. A flattening of the rate of increase of PDE is observed as pulse widths approach 2 ms. Pulse sequences with multiple peaks and a significant amount of superposition or continuing luminosity account for temporal widths of $>2 \mathrm{~ms}$ and are detected with high efficiency (45\% to $60 \%$ ). 


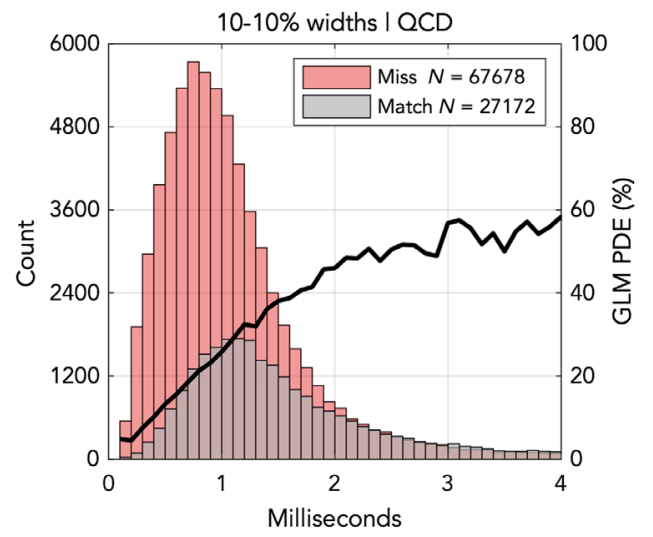

Fig. 8 GLM PDE dependence on the temporal width of the pulse measured at the $10-10 \%$ radiance amplitude. A histogram shows the number of pulses that were measured to have temporal widths in corresponding bins. The histograms are parsed between GLM misses (red) and matches (gray) and scales with the left axis. The PDE is calculated in each width bin and shown as a black trace that scales with the right axis.

\subsection{GLM on Orbit Radiance Detection Threshold}

As a secondary objective, FEGS was deployed to offer an independent verification of the on orbit radiance detection threshold of GLM. An important factor to consider when comparing the two instruments is that FEGS observes a 10-nm wide optical passband. The FEGS measurement offers no information about the spectral distribution of energy within the filter passband, however, measurements by Ref. 18 and others have shown that virtually all of the emission in the FEGS passband is produced by an OI emission triplet that is no more than $1 \mathrm{~nm}$ in width. In contrast, both LIS and GLM utilize filter passbands that are $1 \mathrm{~nm}$ wide and designed to efficiently transmit the OI triplet. Because the lightning signature is emitted from a single, narrow emission band, we are able to appropriately compare radiance values observed by FEGS to similar results from satellite instruments by assuming an effective bandwidth of $1 \mathrm{~nm}$ for the FEGS observations.

We can estimate the minimum cloud-top $J_{\lambda}$ that a lightning discharge must produce in order to be detected by GLM by analyzing FEGS pulse $J_{\lambda}$ distributions. Because the ER-2 is in continuous motion relative to the storm and FEGS has a limited spatial footprint, the radiometric observations represent a random sampling of illuminated cloud-top area produced by each discharge, and the brightest region of a pulse area may lie outside of the FEGS FOV. Considerations of this viewing geometry are discussed in more detail in Sec. 5.3. To increase the likelihood that FEGS observed the maximum radiance produced by the pulse, we analyzed a subset of pulses that had a corresponding detection and location in the ground network (GN) datasets that was $<5 \mathrm{~km}$ offset from the nadir position of the FEGS array. Histograms of the $J_{\lambda}$ distribution for this subset of data are shown in Fig. 9 and are separated into populations of GLM misses and matches. We calculate and plot the PDE in each $J_{\lambda}$ bin as the black trace in Fig. 9 and observe a $50 \%$ PDE at a cloud-top $J_{\lambda}$ value of $9 \mu \mathrm{J} / \mathrm{m}^{2} \mathrm{srnm}$ :

$$
\phi=J_{\lambda} A \Delta \lambda_{e} \Omega
$$

To facilitate a comparison between the $J_{\lambda}$ threshold estimated above and the corresponding radiant energy, $\phi(\mathrm{J})$ that would be incident on a GLM pixel, we use Eq. (1). Here we make the simplifying assumption that all observed lightning is located on the optical axis of the GLM telescope. We also assume that the observed cloud-top $J_{\lambda}$ is uniform over a nominal GLM pixel footprint area $A$ of $64 \mathrm{~km}^{2}$. Then we multiply $J_{\lambda}$ by the $1-\mathrm{nm}$ effective spectral bandwidth of the FEGS radiometer $\Delta \lambda_{e}$. To estimate the solid angle $\Omega$, which is subtended by the GLM entrance aperture as viewed from the cloud-top, we use the small angle approximation shown in the following equation: 


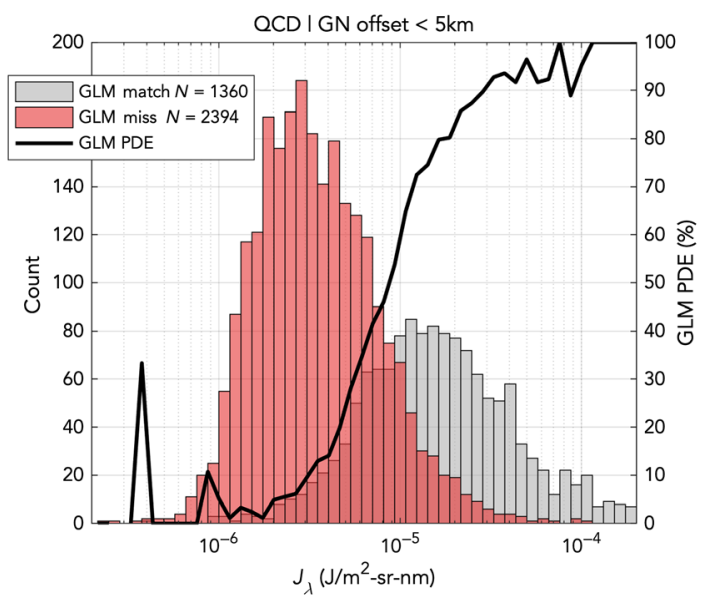

Fig. 9 GLM PDE as a function of $J_{\lambda}$ for pulses with a corresponding GN location that was $<5 \mathrm{~km}$ offset from the FEGS nadir position. The distribution of pulses with a GLM match are shown in gray, and GLM misses are shown in red. The number of pulses in each $J_{\lambda}$ bin scales with the left axis. The black trace indicates the GLM PDE calculated for each $J_{\lambda}$ bin and scales with the right axis. The $50 \%$ PDE occurs at $9 \mu \mathrm{J} / \mathrm{m}^{2} \mathrm{srnm}$.

$$
\Omega=\frac{A_{\text {aperture }}}{d^{2}}
$$

The diameter of the GLM entrance aperture is $112 \mathrm{~mm}$, with a corresponding circular area of $9.85 \times 10^{-3} \mathrm{~m}^{2}$. The distance $d$ between the aperture and Earth in geostationary orbit is about $36,000 \mathrm{~km}$. The resulting solid angle $\Omega$ subtended by the aperture as viewed from the cloud-top is $7.6 \times 10^{-18} \mathrm{sr}$. We estimate that true off axis viewing geometry and variable GLM pixel pitch will give rise to an error as large as $12 \%$ in the $\phi$ estimates below.

From Fig. 9, we find the energy corresponding to the 50\% GLM PDE to be $9 \mu \mathrm{J} / \mathrm{m}^{2} \mathrm{sr} \mathrm{nm}$. Following the conversion above, we estimate a respective $\phi$ value of 4.4 fJ. Figure 10 shows the distribution of detected GLM event energy in the reprocessed dataset that encompasses

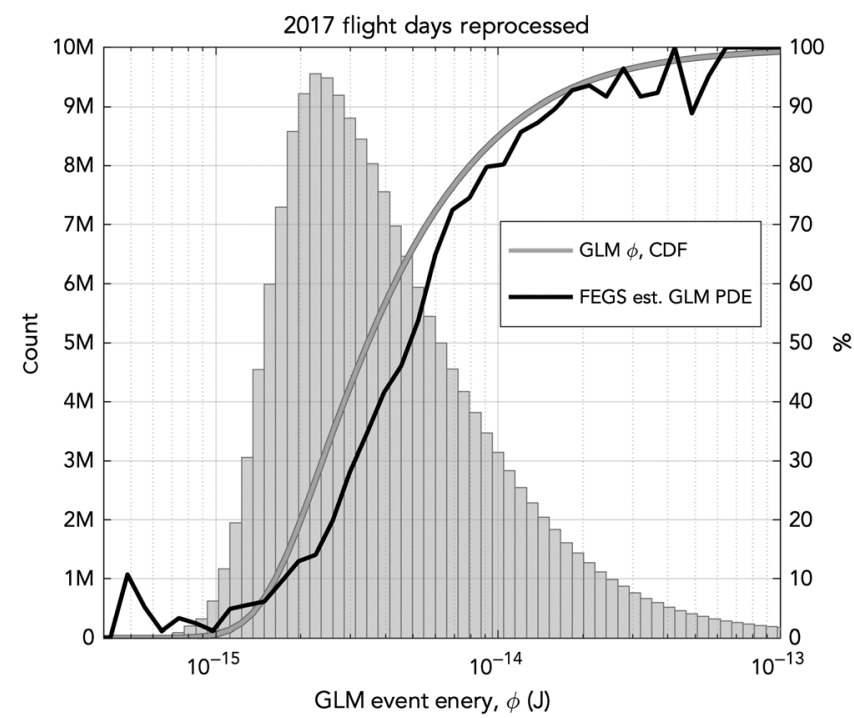

Fig. 10 Distribution of GLM event energy. $\phi$ is the energy in joules incident on a GLM pixel caused by a detected event. The gray histogram shows number distribution of $\phi$ in the reprocessed GLM dataset encompassing field campaign flight days and scales with the left axis. The corresponding cumulative distribution is shown with a gray trace that scales with the right axis. The black trace is the estimated GLM PDE curve from Fig. 9 converted to corresponding $\phi$ as discussed in Sec. 4.3 and scales with the right axis. 
the 2017 field campaign flight days. The events occurred over the full Earth disk and have a median $\phi$ of $3.5 \mathrm{fJ}$. The cumulative distribution of $\phi$ is shown as a solid gray line that scales with the right axis. Finally, on the right axis in Fig. 10, we overlay the estimated GLM PDE curve from Fig. 9 after converting the radiance values as described above.

The PDE curve shown in Figs. 9 and 10 represents an average of GLM detection behavior over the range of locations and time of day observed during the field campaign. We see in the reprocessed dataset that $\sim 65 \%$ of reported GLM events have an energy $<4.4 \mathrm{fJ}$ and FEGS estimates that on average GLM is only detecting $50 \%$ of optical pulses that produce that energy. We would expect GLM to detect a higher percentage of events with this energy near the center of the GLM FOV where radiance threshold settings are typically lower. A $\phi$ value of $1.5 \mathrm{fJ}$ corresponds to a $10 \%$ GLM PDE and an estimated cloud-top $J_{\lambda}$ of $3 \mu \mathrm{J} / \mathrm{m}^{2} \mathrm{srnm}$. This energy, as seen in Fig. 10, corresponds to the center of the low-amplitude drop off in the GLM event number distribution suggesting this value approaches the minimum radiance detection of the instrument.

Because FEGS detects the instantaneous radiance field, its ability to detect a pulse depends on the pulse peak amplitude and not on the temporal width. Therefore, there is no meaningful minimum detectable $J_{\lambda}$ for the FEGS instrument. In the context of GLM validation, however, we can estimate this value $J_{\lambda \text {, min }}$, using the following equation:

$$
J_{\lambda, \min }=6 \times L_{\lambda, \mathrm{bg}, \mathrm{rms}} \times \operatorname{median}\left(\frac{J_{\lambda}}{L_{\lambda, p k}}\right) .
$$

Here $L_{\lambda, \text { bg,rms }}$ denotes the root-mean-square amplitude of the noise signature in the background radiance baseline and is equal to $3.7 \times 10^{-4} \mathrm{~W} / \mathrm{m}^{2} \mathrm{sr} n \mathrm{~nm}$ for the 2017 FEGS observations. A pulse is detected in the version 2 algorithm if its peak radiance is at least 6 times as large as $L_{\lambda, \mathrm{bg}, \mathrm{rms}}$. We multiply that value by the median ratio between the pulse $J_{\lambda}$ and peak radiance $L_{\lambda, p k}$ for pulses in the QCD dataset. The median ratio is equal to $4.5 \times 10^{-4} \mathrm{~J} / \mathrm{W}$, leading to a $J_{\lambda, \text { min }}$ estimate of $9.9 \times 10^{-7} \mathrm{~J} / \mathrm{m}^{2} \mathrm{sr} \mathrm{nm}$. This value converts to an estimated GLM pixel energy of $4.5 \times 10^{-16} \mathrm{~J}$, well below the smallest values reported by GLM. In practice, FEGS can detect pulses with less energy if the pulse is sharply peaked and narrow in time.

\subsection{Spatial Trends of Cloud-Top Illumination and GLM Detection}

We observe a strong correlation between GLM PDE and illuminated cloud-top area. A histogram showing the distribution of estimated area for pulses that were detected by FEGS and the corresponding GLM PDE calculated in bins of $5 \mathrm{~km}^{2}$ is shown in Fig. 11. The maximum area

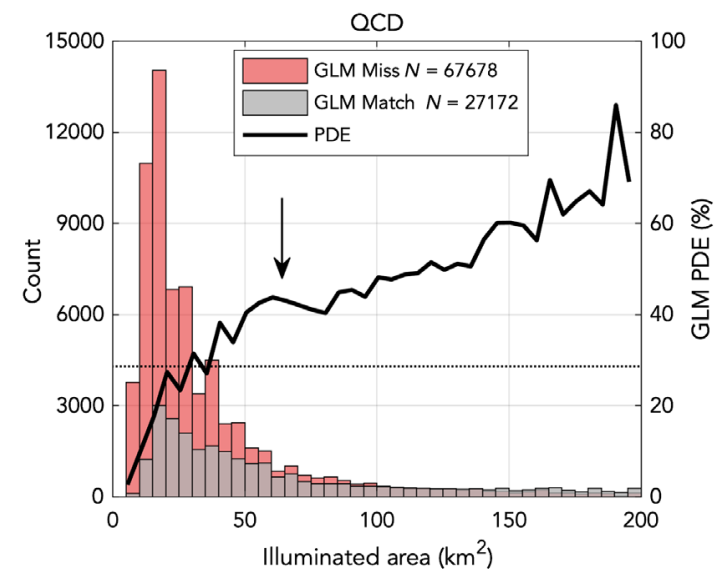

Fig. 11 Histogram showing the distribution of cloud-top area that was illuminated by optical pulses detected by FEGS. The gray bars show the number of pulses that had a corresponding GLM detection, the red bars show the pulses that GLM did not detect, and the black curve shows the PDE in each $5 \mathrm{~km}^{2}$ bin. The arrow is positioned at $64 \mathrm{~km}^{2}$, the nominal footprint of a GLM pixel. 
measured is $196 \mathrm{~km}^{2}$ and corresponds to a fully illuminated FEGS array when FEGS is $7 \mathrm{~km}$ above cloud-top, which was the most common viewing geometry during the GOES-R field campaign. The data are parsed into pulses with a GLM match (gray) and pulses that GLM missed (red). The black trace shows the PDE, and an arrow is positioned at $64 \mathrm{~km}^{2}$, the nominal GLM pixel footprint area. We observe a general increase in PDE with pulse area, and note that there are two apparent regions in the PDE trend defined by a knee in the curve at approximately the location of the arrow in Fig. 11. The rate of increase of PDE with area smaller than a GLM pixel footprint is twice the rate of increase with area larger than a GLM pixel. Figure 11 provides compelling evidence that GLM has difficulty detecting sources that underfill a pixel. Complications associated with the interpretation of this trend related to the FEGS viewing geometry are discussed in Sec. 5.

\section{Discussion}

\subsection{Radiance Detection Threshold Performance Specification}

In Fig. 9, we observe a gradual increase of PDE over the time-integrated spectral radiance decade of $10^{-4}$. The overlap in distributions of GLM misses and matches can be caused by a number of factors including differing viewing geometry between the two instruments, non-uniformity of cloud-top illumination on a sub-GLM-pixel scale, and threshold settings that are dynamically dependent on background solar radiance. More importantly the gradient represents a nonuniform range of GLM threshold settings across the sensor FOV and within the range of satellite boresight angles observed by FEGS during the field campaign.

In order for GLM to detect a lightning flash, it must necessarily detect at least one optical pulse produced by the flash. Presumably GLM would preferentially detect the most energetic pulses. By plotting the distribution of $J_{\lambda}$ for the most energetic pulse in each flash, we can determine the necessary GLM detection threshold to meet a given performance standard based on FDE. Figure 12 shows a frequency distribution (left axis) of maximum $J_{\lambda}$ in the QCD FEGS dataset along with the corresponding CDF (right axis). According to the FEGS measurements, $70 \%$ of lightning flashes produce a maximum cloud-top $J_{\lambda}>1.8 \mu \mathrm{J} / \mathrm{m}^{2} \mathrm{srnm}$. Following the same derivation presented in Sec. 4.3, this corresponds to a radiant energy threshold of $0.9 \times$ $10^{-15} \mathrm{~J}$ incident onto a GLM pixel. Figure 12 also shows the CDF of the subset of FEGS flashes that had a corresponding trigger in the EFCM indicating a substantial electrostatic discharge. The $70 \%$ thresholds in the EFCM subset are larger by a factor of 1.4 with a corresponding GLM pixel energy of $1.3 \times 10^{-15} \mathrm{~J}$.

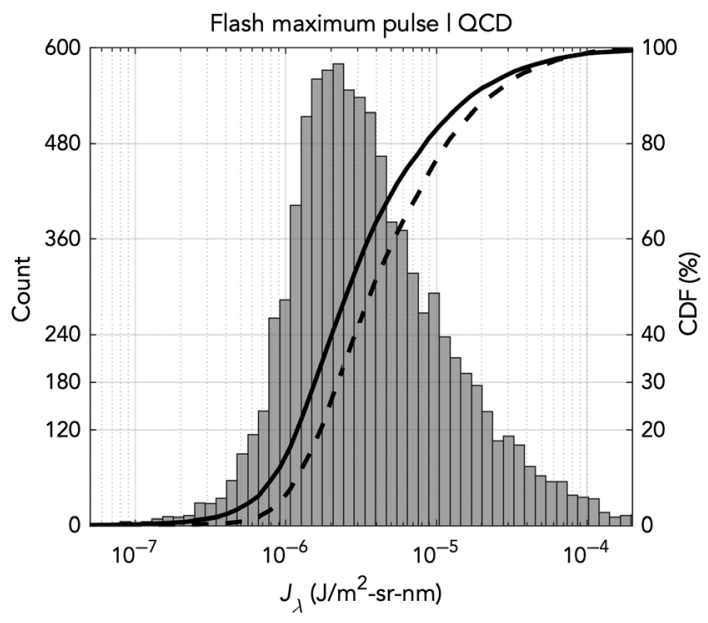

Fig. 12 Distribution of the radiance of the most energetic pulse produced by flashes in the FEGS QCD dataset. The CDF is shown as a solid black trace and scales with the right axis. The dashed trace shows the CDF for the subset of flashes with a simultaneous detection by the EFCM instrument. 
We can compare our threshold values from Sec. 4.3 to similar calculations made by Ref. 26 who estimated minimum detectable cloud-top energy densities $\left(\mathrm{J} / \mathrm{km}^{2}\right)$ for LIS and GLM. For this conversion, we multiply our FEGS $J_{\lambda}$ values by the 1-nm effective bandwidth of the sensor and assume the angular distribution of cloud-top radiance is Lambertian over the upper hemisphere. Our $J_{\lambda}$ values corresponding to $10 \%$ and $50 \%$ GLM PDE, 3 and $9 \mu \mathrm{J} / \mathrm{m}^{2} \mathrm{sr} \mathrm{nm}$, respectively, convert to an energy density of 9 and $28 \mathrm{~J} / \mathrm{km}^{2}$. These values are larger than the $6.6-\mathrm{J} / \mathrm{km}^{2}$ minimum detectable cloud-top energy density reported by Ref. 26 as the most common threshold in the central 1/3 of the GLM FOV. We find good agreement in the values considering the FEGS measurements were taken at larger satellite boresight angle where GLM threshold settings are higher by a factor of 2 to 4 .

In the context of FEGS observations, a flash is defined as a temporally isolated series of optical pulses. With the relatively high sensitivity of the instrument, FEGS will detect light from in-cloud processes that may not fully manifest into high-current discharges (e.g., a stroke) that more appropriately define a lightning flash. Failed leader attempts are an example of a process that FEGS is likely to observe but that GLM was not designed to detect. As a result, the FEGS observations include a population low-amplitude processes that lead to a lower bound estimate of GLM FDE. If a subset of FEGS flashes with a simultaneous trigger on the slow E-field channel of the EFCM (indicating a significant electrostatic discharge) are analyzed the average daytime GLM FDE increases to 76\%. A more in-depth analysis of the combined FEGS and EFCM signatures is warranted to provide insight into the GLM DE for varying lightning processes.

As an aside, we note that due to limitations in the NOAA data processing ground system, the first pulse (group) of a GLM flash will be discarded unless the lightning cluster filter algorithm ${ }^{20}$ has been activated in proximity to the group within the prior 1 -s interval. Although the detected first group may exist in the original level 0 data, it will not be transmitted through to the level 2 output. This will artificially reduce the DE of GLM for singlepulse flashes and is likely the cause of the delayed flash onset shown in Fig. 7. We recommend for the development of a set of "scientific" data processing algorithms in order to maximize the value of GLM.

\subsection{Relationship Between Pulse Area and Pulse Radiance}

We find a correlation between the pulse area and the pulse radiance in the FEGS dataset and illustrate the relationship in Fig. 13. When the mean (and median) of $J_{\lambda}$ is calculated for pulses binned by area in increments of $5 \mathrm{~km}^{2}$, we find a positive correlation where increasing $J_{\lambda}$ corresponds to increasing illuminated area. A second manifestation of this relationship is realized when the average area and radiance are calculated independently as a function of time over the

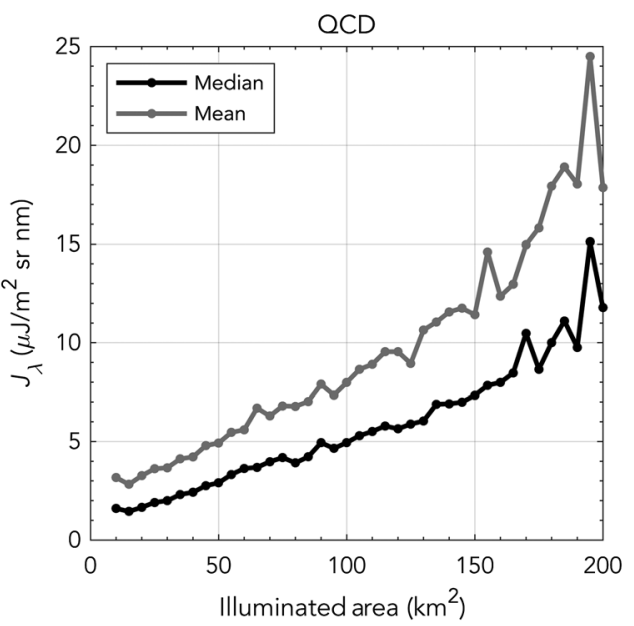

Fig. 13 Illuminated cloud-top area verses radiance for optical pulses detected by FEGS. The plot shows the mean and median $J_{\lambda}$ of pulses with area binned in $5 \mathrm{~km}^{2}$ increments. 
duration of a flash. Both parameters are observed to increase on average as flashes evolve. This relationship presents a doubly difficult scenario for GLM detection of small (and dim) pulses and leads to the most likely explanation of the positive correlation between GLM FDE and flash duration (see Fig. 6). Specifically, as flash durations grow longer, the production of a bright, large area pulse becomes more probable, leading to a higher GLM FDE. These findings and our interpretation are consistent with the observations and inferences reported by Ref. 26. As a precaution in interpreting these trends, however, it is important to thoroughly consider the constraints of the FEGS viewing geometry.

\subsection{Geometrical Considerations}

Although FEGS can provide higher spatial resolution than GLM of cloud-top radiance distributions, the FEGS observation is constrained to a limited spatial footprint. The distribution of lateral distance offset between the FEGS position and the GLM flash centroid stated in Sec. 4.1 is a result of the spatial extent of GLM flashes and the random sampling nature of the FEGS aircraft observation. Figure 14 shows a map projection of a lightning flash that was detected coincidently by both FEGS and GLM. In this example, analysis of the FEGS detection would indicate that 4 pixels were illuminated with a resulting cloud-top area estimation of $28 \mathrm{~km}^{2}$. Of course, the full spatial extent of the flash as observed by GLM is much larger, $911 \mathrm{~km}^{2}$. Additionally, FEGS is displaced $8.9 \mathrm{~km}$ from the flash centroid and is positioned on the outer edge of the lobe of illuminated cloud top. It is expected that this area would have a reduced radiance corresponding to the tail of a Gaussian like distribution. In fact, there is a clear trend in the FEGS dataset indicating a decrease in both the number of pixels illuminated and the observed pulse $J_{\lambda}$ with increasing offset distance between the GLM flash centroid and the FEGS location at the time of pulse detection. This constraint of the aircraft observation may hinder the ability to make a reliable determination of relationships between pulse area and pulse radiance. Specifically, the positive correlation between the two parameters highlighted in Sec. 4.4 could be interpreted as the result of the viewing geometry shown in Fig. 14.

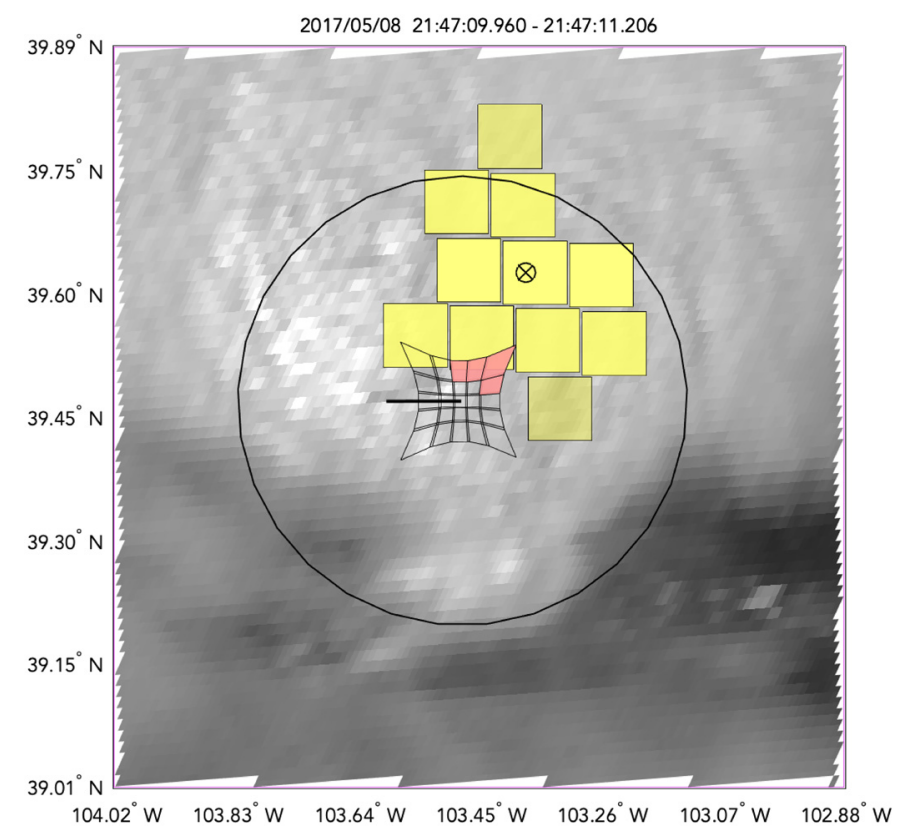

Fig. 14 Map projection of a lightning flash that was coincidently detected by both GLM and FEGS. The GLM pixel locations are shaded in yellow and the flash centroid is marked by a circled $X$. The projected FEGS FOV is shown with illuminated pixels shaded red. A ring illustrates the $30-\mathrm{km}$ offset distance that was used as a spatial constraint for the matching comparison between the two datasets. The line extending from the center of the FEGS FOV indicates the direction of aircraft motion. The background image is visible cloud imagery from GOES-15. 
(a)

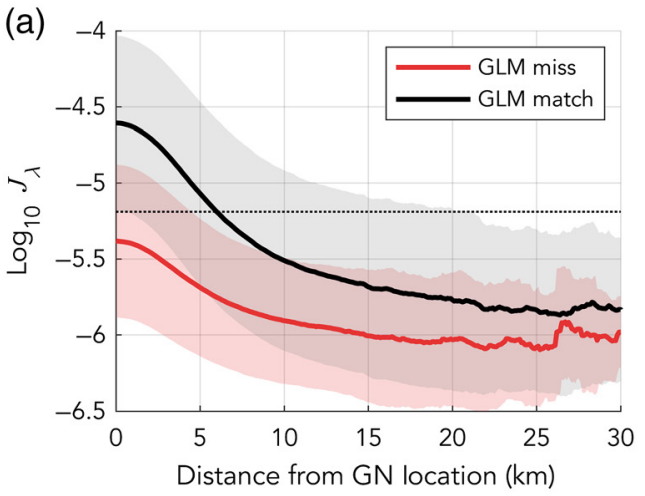

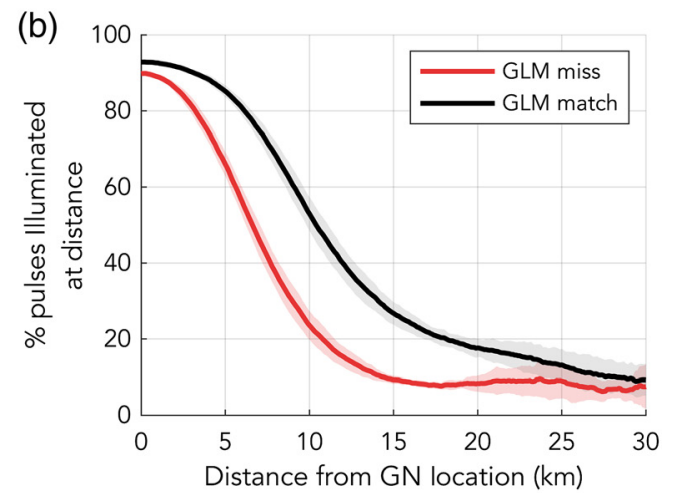

Fig. 15 Accumulated average radiance spatial distribution radial profile for optical pulses detected by FEGS that GLM missed (red) or matched (black). (a) The amplitude of the log of radiance as a function of distance from the GN location. (b) The number of pulses that produced light at a given distance from the GN location.

To test the reliability of our interpretation, we performed an experiment with the FEGS data to accumulate a geometrically averaged cloud-top $J_{\lambda}$ distribution. The analysis included the subset of FEGS pulses that had a corresponding match in the GN datasets to provide a lat/lon location for the discharge. The FEGS FOV was then oriented with respect to the ground location on a $40 \times 40 \mathrm{~km}$ grid with $0.1 \mathrm{~km}$ resolution. $J_{\lambda}$ values that were detected in each grid cell were accumulated and then averaged over the number of pulses that were detected in the grid cell. The dataset was parsed into pulses with a GLM match and pulses that GLM missed. The process produced a Gaussian shaped $J_{\lambda}$ distribution for both categories of pulses and the axially symmetric radial profiles are shown in Fig. 15. The left panel of Fig. 15 shows the distribution of the $\log$ of $J_{\lambda}$ for GLM misses and matches. Pulses that GLM detected exceeded the estimated $J_{\lambda}$ detection threshold (see Sec. 4.3), shown as a dotted line in the plot, more often and over a larger area than did the pulses that GLM did not detect.

Figure 15(b) shows the percentage of pulses that produced light at a given distance from the ground contact location out of the number of times FEGS sampled at that distance. It is interesting here to note that $<100 \%$ of pulses produce light at the cloud-top directly above the GN location. This curve illustrates that pulses that were detected by GLM illuminate a larger average area than pulses that GLM does not detect. In fact, of the missed pulses, only 50\% were observed to have light emissions at a distance of $6.6 \mathrm{~km}$ from the GN location, whereas the $50 \%$ threshold for matches is $10.4 \mathrm{~km}$. If we assume that the lobe of illumination at the cloud-top is circular, we find that the median area of matched pulses is 2.5 times larger than the median of missed pulses. These statistical results lend further support to our interpretation of the positive correlation between area and radiance discussed in Sec. 5.2.

In the extremity of FEGS viewing geometry, as the ER-2 approaches or departs a storm, FEGS will begin to detect low-amplitude illuminations that may be caused by flashes in the active region of a storm outside the $30-\mathrm{km}$ spatial matching radius used in this study. The radius was chosen as a statistically optimum distance to avoid mismatching. Indeed, a timeseries of GLM FDE over the course of a FEGS flight shows a repeated decrease in FDE before and after FEGS overflew the electrically active region of storms. This effect will reduce the estimated overall GLM FDE. A recent report by Ref. 27 calculated GLM FDE using FEGS data to study nitrogen oxide production in thunderstorms. They considered a subset of 10-min sample periods when cloud-top height measurements from the Cloud Physics Lidar ${ }^{28}$ indicated the ER-2 was over deep convection. The estimated GLM FDE was $75 \% \pm 16 \%$. Further analysis of individual storm cases is warranted to assess the impact of this observational discrepancy.

\subsection{Small Area Sources}

We have the special case where the entire cloud-top area of a pulse was contained within the FEGS FOV so that only a combination of the inner 9 pixels in the FEGS array were illuminated. This subset of data included 5752 optical pulses (6.1\% of the FEGS QCD dataset). These pulses 
were observed in similar percentages in each flight, fell on the low-amplitude end of the pulse $J_{\lambda}$ distribution, and occurred with regular frequency throughout the duration of flashes. We note that GLM only detected 3.9\% of these pulses. Comparison to GN data indicates that only $6 \%$ of these pulses were detected by GNs and were classified as intracloud (IC) sources $94 \%$ of the time. Peak current estimates of these sources provided by the ground networks fall on the lowamplitude end of the IC distribution. ${ }^{24}$ Initial analysis of EFCM signatures suggest that many of them are produced by $\mathrm{k}$-type processe ${ }^{29}$ associated with negative leader development during the flash.

\section{Conclusions}

The FEGS airborne radiometer array provides a critical validation capability for GLM by observing cloud-top optical emission with greater spatial and temporal resolution and higher sensitivity than GLM. During the GOES-R validation flight campaign FEGS observed over 14,000 lightning flashes during both day and night for a variety of convective scenarios and geographical regions. Of these flashes, a subset of 9160 flashes occurring during daytime hours was found suitable for analysis following the application of a QC process. An analysis of this QCD field campaign dataset indicates that the average daytime FDE of GOES-16 GLM is at least $64 \%$ but varies significantly between storms. The actual GLM FDE is likely $~ 10 \%$ higher if only flashes with high-current strokes are considered. There is a strong dependence of GLM FDE on the duration of a flash caused by an increased likelihood of the production of a large area, bright pulse with an increased flash lifetime. Pulses with longer duration and higher $J_{\lambda}$ amplitudes are more likely to be detected with an upper limit estimated $J_{\lambda}$ detection threshold of $9 \mu \mathrm{J} / \mathrm{m}^{2} \mathrm{sr} \mathrm{nm}$ at the cloud-top. GLM has particular difficulty detecting small area optical pulses that underfill a pixel and on average emit less energy per unit area and solid angle. Initial analysis of electric field waveforms and GN data indicates many of these small area sources are cloud discharge processes with low-current amplitudes.

FEGS has provided a wealth of information regarding the characteristics of the population of optical pulses that are produced by a wide variety of thunderstorm phenomenologies. Further analysis of the dataset is warranted to associate these characteristics to the physical discharge process that produces them, the radiative transfer properties of the intermediate cloud, and the corresponding thunderstorm dynamics. Electric field waveforms provided by the EFCM on the ER-2 as well as GN datasets can be utilized for lightning process discrimination. Intercomparison with lightning mapping array data ${ }^{30}$ to provide an estimate of the lightning channel geometry will facilitate better understanding of the temporal width of optical pulses in relation to the cloud-optical depth. Future FEGS flights with upgraded electronics will provide insight into the day-to-night dynamic performance of GLM as a result of varying background solar radiance conditions.

\section{Acknowledgments}

This work was funded by the NOAA GOES-R Series Science, Demonstration and Cal/Val Program at MSFC under transfers of support from NOAA NESDIS-GOES-R to NASAMSFC (several annual order numbers) and most recently through direct transfer to NOAA CISESS. Additional support was provided by the NASA Postdoctoral Program. The version 2 FEGS dataset collected during the 2017 GOES-R Validation Flight Campaign, as well as the reprocessed GLM data can be found at the website for the Global Hydrology Resource Center: http://dx.doi.org/10.5067/GOESRPLT/DATA101

\section{References}

1. M. Brook et al., "Simultaneous observations of lightning radiations from above and below clouds," Geophys. Res. Lett. 7(4), 267-270 (1980).

2. M. Brook et al., "Nighttime observations of thunderstorms electrical activity from a high altitude airplane," J. Geophys. Res. 90(D4), 6111-6120 (1985). 
3. H. J. Christian et al., "Observations of optical lightning emissions from above thunderstorms using U-2 aircraft," Bull. Am. Meteorol. Soc. 64(2), 120-123 (1983).

4. H. J. Christian and S. J. Goodman, "Optical observations of lightning from a high-altitude airplane," J. Atmos. Oceanic Technol. 4, 701-711 (1987).

5. S. J. Goodman, H. J. Christian, and W. D. Rust, "A comparison of the optical pulse characteristics of intracloud and cloud-to-ground lightning as observed above clouds," J. Appl. Meteor. 27, 1369-1381 (1988).

6. H. J. Christian et al., "Global frequency and distribution of lightning as observed from space by the optical transient detector," J. Geophys. Res. 108(D1), ACL 4-1-ACL 4-15 (2003).

7. H. J. Christian, R. J. Blakeslee, and S. J. Goodman, "Lightning imaging sensor for the earth observing system," Tech. Rep. NASA TM-4350, NASA, Washington, DC (1992).

8. D. J. Boccippio, W. J. Koshak, and R. J. Blakeslee, "Performance assessment of the optical transient detector and lightning imaging sensor. Part I: predicted diurnal variability," J. Atmos. Oceanic Technol. 19, 1318-1332 (2002).

9. R. J. Blakeslee and W. Koshak, "LIS on ISS: expanded global coverage and enhanced applications," The Earth Observer 28, 4-14, 2016, http://eospso.nasa.gov/sites/default/files/ eo_pdfs/May_June_2016_color\%20508.pdf\#page=4.

10. R. J. Blakeslee et al., "Three years of the lightning imaging sensor onboard the International Space Station: expanded global coverage and enhanced applications," J. Geophys. Res. Atmos. 125, e2020JD032918 (2020).

11. D. M. Mach et al., "Lightning optical pulse statistics from storm overflights during the Altus Cumulus Electrification Study," Atmos. Res. 76, 386-401 (2005).

12. S. J. Goodman et al., "The GOES-R geostationary lightning mapper (GLM)," Atmos. Res. 125-126, 34-49 (2013).

13. S. D. Rudlosky et al., "Initial geostationary lightning mapper observations," Geophys. Res. Lett. 46, 1097-1104 (2019).

14. T. J. Schmit et al., "Geostationary operational environmental satellite (GOES)-14 super rapid scan operations to prepare for GOES-R," J. Appl. Remote Sens. 7(1), 073462 (2013).

15. F. Padula et al., "GOES-R advanced baseline imager (ABI) and geostationary lightning mapper (GLM) calibration/validation from a field campaign perspective," in IEEE Int. Geosci. and Remote Sens. Symp., Fort Worth, Texas, pp. 301-304 (2017).

16. K. Thompson, M. Bateman, and L. Carey, "A comparison of two ground-based lightning detection networks against the satellite-based lightning imaging sensor (LIS)," J. Atmos. Oceanic Technol. 31, 2191-2205 (2014).

17. R. E. Orville and R. W. Henderson, "Absolute spectral irradiance measurements of lightning from 375 to 880 nm," J. Atmos. Sci. 41, 3180-3187 (1984).

18. T. D. Walker and H. J. Christian, "Triggered lightning spectroscopy: part 1. A qualitative analysis," J. Geophys. Res. Atmos. 122, 8000-8011 (2017).

19. N. Østgaard et al., "Gamma-ray glow observations at $20 \mathrm{~km}$ altitude," J. Geophys. Res. 124(13), 7236-7254 (2019).

20. S. Goodman et al., "GLM lightning cluster filter algorithm," NOAA NESDIS Center for Satellite Applications and Research, Algorithm Theoretical Basis Document, Version 3.0, 2012, https://www.star.nesdis.noaa.gov/goesr/documents/ATBDs/Baseline/ATBD_GOESR_GLM_v3.0_Jul2012.pdf.

21. D. M. Mach, "Geostationary lightning mapper clustering algorithm stability," J. Geophys. Res. Atmos. 125(5), e2019JD031900 (2020).

22. R. J. Blakeslee et al., "Seasonal variations in the lightning diurnal cycle and implications for the global electric circuit," Atmos. Res. 135-136, 228-243 (2014).

23. "GOES-R algorithm working group and GOES-R series program," Product Definition and User's Guide, Volume 3: Level 1b Products, DCN 7035538, Revision F.1, 2017, https:// www.goes-r.gov/users/docs/PUG-L1b-vol3.pdf.

24. A. Nag et al., "Recent evolution of the U.S. National Lightning Detection Network," in 23rd Int. Lightning Detection Conf. (2014).

25. V. Bui, L. C. Chang, and S. Heckman, "A performance study of earth networks total lightning network (ENTLN) and worldwide lightning location network (WWLLN)," in Int. Conf. Comput. Sci. and Comput. Intell., Las Vegas, Nevada (2015). 
26. D. Zhang and K. L. Cummins, "Time evolution of satellite-based optical properties in lightning flashes, and its impact on GLM flash detection," J. Geophys. Res. Atmos. 125, e2019JD032024 (2020).

27. D. J. Alle et al., "Observations of lightning NOx production from GOES-R post launch test field campaign flights," J. Geophys. Res. Atmos. in review (2020).

28. M. McGill et al., "GOES-R PLT Cloud Physics LiDAR (CPL)," NASA Global Hydrology Resource Center DAAC, Huntsville, Alabama, 2019, http://dx.doi.org/10 .5067/GOESRPLT/CPL/DATA101.

29. V. A. Rakov, R. Thottappillil, and M. A. Uman, "Electric field pulses in $\mathrm{K}$ and $\mathrm{M}$ changes of lightning ground flashes," J. Geophys. Res. 97(D9), 9935-9950 (1992).

30. R. J. Thomas et al., "Accuracy of the lightning mapping array," J. Geophys. Res. 109, D14207 (2004).

Mason G. Quick is an atmospheric physicist and has utilized a variety of remote sensing technologies to study thunderstorm electricity. His experience with radio frequency and optical remote sensing instrumentation couples with comprehensive knowledge of atmospheric sciences to provide a well-rounded and thorough understanding of the field. He served as the lead scientist in the design, calibration, and operation of the Fly's Eye GLM Simulator as part of the GOES-R validation effort.

Hugh J. Christian received his $\mathrm{PhD}$ in space physics and astronomy from Rice University. After a postdoc, he joined NASA's MSFC where he founded a lightning research group. He was PI on the optical transient detector and the lightning imaging sensor and designed the geostationary lightning mapper. Upon retiring from NASA, he joined the University of Alabama Huntsville where he helped develop high-speed lightning spectroscopy, electric field change networks, and aircraft electric filed measurements.

Katrina S. Virts received her $\mathrm{PhD}$ in atmospheric science from the University of Washington and is currently a research scientist at the University of Alabama in Huntsville. She is a member of the calibration/validation team for the geostationary lightning mapper and is also part of NASA's Interagency Implementation and Advanced Concepts Team. Her research interests include lightning climatology, diurnal effects, mesoscale convection, and tropical meteorology.

Richard J. Blakeslee is a senior research scientist and expert in lightning and atmospheric electricity research and its applications. For more than four decades, he has pursued satellite, airborne, and ground-based lightning and storm observations to make important contributions to fundamental Earth science questions in weather, climate, atmospheric chemistry, and lightning physics. He is an author and co-author of numerous nationally and internationally recognized scientific articles documenting the results of his research and collaborations. 